\title{
Predictive model of magnetosheath plasma flow and its validation against Cluster and THEMIS data
}

\author{
J. Soucek ${ }^{1,2}$ and C. P. Escoubet ${ }^{1}$ \\ ${ }^{1}$ ESTEC, European Space Agency, Keplerlaan 1, 2201 AZ, Noordwijk, The Netherlands \\ ${ }^{2}$ Institute of Atmospheric Physics, Bocni II 1401, 14131 Prague, Czech Republic
}

Correspondence to: J. Soucek (soucek@ufa.cas.cz)

Received: 19 March 2012 - Revised: 25 May 2012 - Accepted: 27 May 2012 - Published: 12 June 2012

\begin{abstract}
An analytical model of magnetosheath plasma flow is described and compared with a large dataset of magnetosheath ion flow velocity measurements from Cluster and THEMIS spacecraft. The model is based on previous works by Kobel and Flückiger (1994) and Génot et al. (2011) and has been modified to overcome the restrictions of these models on the shape of model magnetopause and bow shock. Our model is compatible with any parabolic bow shock model and arbitrary magnetopause model. The model is relatively simple to implement and computationally inexpensive, and its only inputs are upstream solar wind parameters. Comparison with observed data yields a good correspondence: median error in the direction of flow velocity is comparable with the instrumental error, and flow magnitude is predicted with a reasonable accuracy (relative error in flow speed was less than $25 \%$ for $86.5 \%$ of observations).
\end{abstract}

Keywords. Magnetospheric physics (Magnetosheath)

\section{Introduction}

Modeling of the flow of solar wind plasma around the magnetosphere of the Earth has a long history reaching back to the dawn of space exploration. After the supersonic solar wind is decelerated and heated at the Earth's bow shock, it flows almost laminarly around the magnetospheric cavity, forming the region known as the magnetosheath. A good magnetosheath flow model is required in a wide range of space plasma studies. In the context of interpretation of in situ spacecraft data, such a model can be applied to trace the flow of magnetosheath plasma from the point of observation inside the magnetosheath (location of the spacecraft) to the point of origin of the same flowline at the bow shock (for example Tátrallyay and Erdős, 2002; Tátrallyay et al., 2008; Génot et al., 2011; Hayosh et al., 2005) or to trace the plasma flow between two spacecraft. The model then provides information on the history of the observed plasma and also on the heating process at the bow shock. The properties of the shock heating depend significantly on local shock parameters, such as the angle between the shock normal and the upstream interplanetary magnetic field (IMF), $\Theta_{B n}$, and the angle between the shock normal and solar wind flow velocity. The exact point of origin at the shock therefore needs to be determined with a reasonable accuracy.

As mentioned for example by Génot et al. (2011), an important property of any magnetosheath velocity model is its practical usability. We set the following criteria for a usable and easily adoptable magnetosheath flow model: (1) It should be relatively easy and computationally inexpensive to implement and adapt to the needs of a specific application. This is particularly important for large statistical studies, where the model needs to be evaluated for every datapoint. (2) All input parameters and initial conditions should be easily accessible. Ideally, measured upstream solar wind parameters and spacecraft position should be the only inputs to the model.

\subsection{Existing magnetosheath models}

The problem has mostly been approached by some form of approximate solution to the gas-dynamic equations governing the flow of unmagnetized fluid around an obstacle represented by the magnetopause. One of the earliest and still widely used models is the hydrodynamic model (Spreiter et al., 1966), which serves as a basis for a complete predictive solar wind-magnetosheath interaction model (Spreiter and Stahara, 1994, 1980; Stahara et al., 1993; Song et al., 1999a) and has also been applied to model the magnetospheres of 
other planets of the solar system (for a review of this class of models, see Stahara, 2002). To our knowledge, this gasdynamic model is the only magnetosheath flow model which has been systematically tested against in situ spacecraft observations. Comparison of the model prediction with measurements from ISEE-2 (Song et al., 1999b; Stahara et al., 1993), Pioneer VI (Spreiter and Alksne, 1968), IMP-1 and other spacecraft (Spreiter et al., 1968) demonstrated a remarkably good correspondence between the model and observations. A major drawback of this model is, however, its computational complexity: each application of the model to a particular set of upstream conditions requires a numerical solution of a set of differential equations on a two- or threedimensional grid.

The magnetosheath flow model proposed in the present paper is based on an analytical model first developed in Kobel and Flückiger (1994) (hereafter KF94 model) and on its extension presented recently in Génot et al. (2011). The original KF94 paper describes an analytical model for the magnetosheath magnetic field, assuming that the IMF does not penetrate inside the magnetosphere and that currents are only present at the bow shock and the magnetopause $(\nabla \times \boldsymbol{B}=0)$. The expressions for the magnetic field are then obtained by solving the Laplace equation for magnetic field potential in parabolic coordinates. The authors themselves mention that for a special case where the IMF is parallel to the solar wind flow, the magnetic field-lines coincide with the plasma flowlines and the KF94 model can thus be used as a magnetosheath flowline model. This model has been exploited in several studies to trace the magnetosheath plasma flowlines (Tátrallyay and Erdős, 2002; Tátrallyay et al., 2008; Génot et al., 2011). In particular, Génot et al. (2011) developed the model further by estimating the downstream flow velocity from Rankine-Hugoniot relations and introducing an ad hoc density model to account for the observed density drop near the magnetopause.

Several other simple models, where the flow velocity vector can be expressed by an analytical formula, have been proposed in the literature. Russell et al. (1983) used a simple analytical expression to approximate the plasma flow streamlines in their study, but no attempts to relate the model to magnetospheric boundaries or to validate the model were made. Kallio and Koskinen (2000) introduced a semiempirical model for the magnetosheath magnetic fields assuming paraboloidal shock and magnetopause and a specific magnetospheric field model. This model is empirical in the sense that its functional form was constructed to resemble the shape of magnetosheath flowlines obtained in numerical simulations and includes several free parameters that can be adjusted to obtain a good fit.

Romashets et al. (2008) developed a more sophisticated analytical model of the solar wind and magnetosheath magnetic field, compatible with Rankine-Hugoniot conditions and basic physical constraints imposed by Maxwell's equations and expected field geometry at the magnetopause. This model is again formulated in parabolic coordinates and imposes strong restrictions on the shape of the magnetospheric boundaries.

In principle these alternative models of magnetosheath flow (Romashets et al., 2008; Kallio and Koskinen, 2000), in their simplified form where only the plasma flow velocity is calculated, could be used in this study instead of KF94. We chose the KF94 model primarily for its simplicity, consistency and tractability. The empirical Kallio and Koskinen (2000) would have to be adjusted by a proper choice or fitting of the free parameters for this purpose. This would introduce an additional empirical element in the model, which we prefer to avoid. The Romashets et al. (2008) model would be more appropriate, being similar to KF94 in the formulation, assumptions and boundary conditions. However, for our application the KF94 formulation is much more tractable, expressing the magnetic field vector (and the flow velocity vector) by relatively simple formulas in Cartesian coordinates using parameters with a direct physical interpretation. Furthermore, since both models start from the same assumptions and use identical shapes of the shock and magnetopause boundaries, the results should be consistent for the special case we use.

With the exception of the gas-dynamic models based on the approach of Spreiter et al. (1966), none of the above models have been systematically tested and validated using spacecraft data. Furthermore, the practical use of the KF94 model (as well as Kallio and Koskinen, 2000, or Romashets et al., 2008) is complicated by the assumed shape of the bow shock and the magnetopause. The model is formulated in parabolic coordinates and both boundaries are expressed as iso-contours. The model thus requires the bow-shock and the magnetopause to be modeled by paraboloids with the same focus. The shape of each boundary is then fully determined by a single parameter (for example the stand-off distance). The choice of bow-shock and magnetosheath models is thus severely restricted, and the acceptable models may not fit the physical boundaries very well. This problem is most severe at the flanks of the magnetosheath; the parabolic approximation is reasonably accurate in the sub-solar magnetosheath but progressively worsens further downstream. This effect is demonstrated in more detail in Sect. 3.2.

In this paper we present a magnetosheath flow model, based on the works of Génot et al. (2011) and Kobel and Flückiger (1994), which overcomes the above difficulties. Our model allows calculating the plasma flow velocity vector for a given point in the magnetosheath using only the spacecraft position and solar wind parameters as an input. The model is compatible with a wide range of bow-shock and magnetopause models and retains the simplicity and computational efficiency of the original models. The model is described in Sect. 2, and its performance is evaluated on a large statistical dataset of velocity measurements from Cluster and THEMIS spacecraft in Sect. 3. 


\section{Description of the model}

The proposed model is formulated in an aberrated GSE coordinate system, obtained by a rotation of the GSE coordinates such that the solar wind flow velocity is aligned with the $\mathrm{x}$-axis. This correction is performed to compensate for the aberration of solar wind flow due to the orbital motion of the Earth. In this corrected coordinate system, the problem is considered cylindrically symmetric around the $\mathrm{x}$-axis. Furthermore, we introduce a spherical coordinate system associated with the aberrated GSE coordinates:

$x=\boldsymbol{r} \cos \vartheta$

$y=\boldsymbol{r} \sin \vartheta \sin \varphi$

$z=r \sin \vartheta \cos \varphi$

where $\vartheta$ is the angle between the vector $\boldsymbol{r}=(x, y, z)$ and the aberrated GSE x-axis, and $\varphi$ is an azimuthal angle in the $y-z$ plane (the angles $\vartheta$ and $\varphi$ correspond to the zenith and clock angles of Verigin et al., 2006).

Our magnetosheath flow model is based on the magnetic field model introduced in Kobel and Flückiger (1994). For clarity, all coordinates and variables associated with the KF94 model are identified by a tilde $(\sim)$. In the KF94, the bow-shock and the magnetopause are modeled by parabolic surfaces with stand-off distances $R_{\mathrm{bs}}$ and $R_{\mathrm{mp}}$ and a common focus at $\boldsymbol{r}_{\mathrm{f}}=\left(R_{\mathrm{mp}} / 2,0,0\right)$ :

$\tilde{x}_{\{\mathrm{bs}, \mathrm{mp}\}}=R_{\{\mathrm{bs}, \mathrm{mp}\}}-b_{\{\mathrm{bs}, \mathrm{mp}\}}\left(y^{2}+z^{2}\right)$

where $b_{\mathrm{bs}}=1 /\left(4 R_{\mathrm{bs}}-2 R_{\mathrm{mp}}\right)$ and $b_{\mathrm{mp}}=1 / 2 R_{\mathrm{mp}}$. The above can be rewritten in spherical coordinates as

$\tilde{r}_{\{\mathrm{bs}, \mathrm{mp}\}}=\frac{-\cos \vartheta+\sqrt{\cos ^{2} \vartheta-4 R_{\{\mathrm{bs}, \mathrm{mp}\}} b_{\{\mathrm{bs}, \mathrm{mp}\}} \sin ^{2} \vartheta}}{2 b_{\{\mathrm{bs}, \mathrm{mp}\}} \sin ^{2} \vartheta}$.

In a special case where the IMF is parallel to the solar wind flow velocity, the magnetic field lines also represent the flowlines of solar wind and magnetosheath plasma. Note that this analogy between the flow velocity direction and magnetic field vector can only be used for the direction of the vectors and the same does not apply to the relative magnitude. Using this property, the KF94 flow velocity vector at a point $\boldsymbol{r}=(x, y, z)$ can be written as:

$\tilde{v}_{\mathrm{x}}=v_{m 0}\left(C / 2 d-C / R_{\mathrm{mp}}\right)$

$\tilde{v}_{\mathrm{y}}=v_{m 0} C y /\left[2 d\left(d+x-x_{\mathrm{f}}\right)\right]$

$\tilde{v}_{\mathrm{z}}=v_{m 0} C z /\left[2 d\left(d+x-x_{\mathrm{f}}\right)\right]$

where $v_{m 0}$ is a positive constant linked to the magnitude of the flow velocity (not addressed by the original KF94 model, which will be discussed in Sect. 2.2), $\boldsymbol{r}_{\mathrm{f}}=\left(x_{\mathrm{f}}, 0,0\right)$ the common focus of the paraboloid surfaces, $d=\left|\mathbf{r}-\mathbf{r}_{\mathrm{f}}\right|$ and $C=R_{\mathrm{mp}}\left(2 R_{\mathrm{bs}}-R_{\mathrm{mp}}\right) /\left(2 R_{\mathrm{bs}}-2 R_{\mathrm{mp}}\right)$. Equations (3) are easily obtained from expressions (32)-(37) in Kobel and

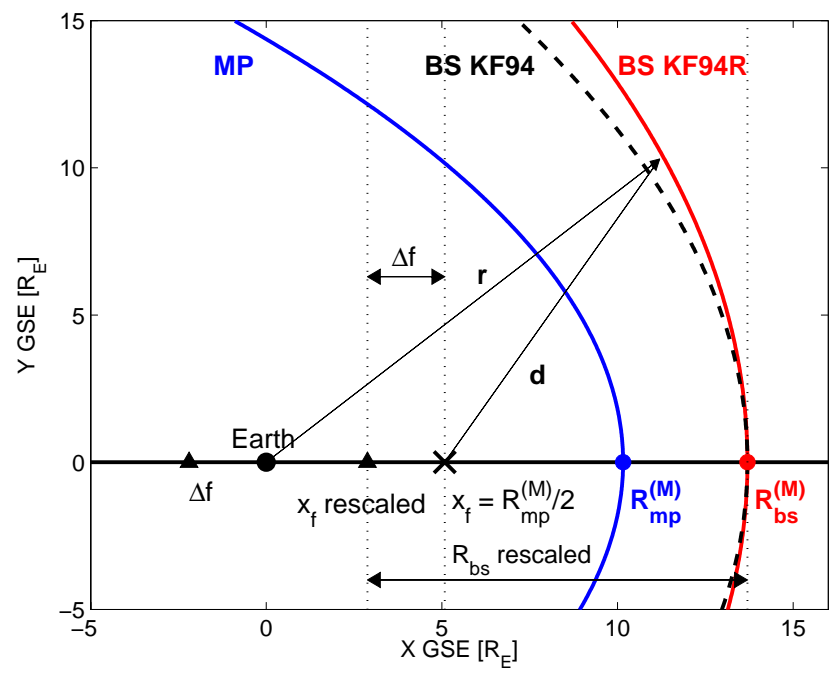

Fig. 1. A schematic representation of the parameters used in the formulation of the KF94 model and in rescaling from KF94 to KF94R. Note that $\Delta f$ is negative in this case, as the focus $x_{\mathrm{f}}$ is being shifted anti-sunward to decrease the curvature of the model surface.

Flückiger (1994) by substituting for their $B^{\mathrm{IMF}}$ a vector parallel to the GSE $\mathrm{x}$-axis, in our case $\left(-v_{m 0}, 0,0\right)$. The geometry of the model and its parameters are schematically depicted in Fig. 1. Note that the reference paper uses a different Cartesian coordinate system with the origin at the focus of the parabolas, where the $-\mathrm{Z}$ axis coincides with GSE $+\mathrm{X}$ axis.

\subsection{Rescaling the KF94 model}

As mentioned previously, a major drawback of the KF94 model is the fixed shape of the bow shock and magnetopause given by Eq. (2), where the "flaring" parameter $b_{\{\mathrm{bs}, \mathrm{mp}\}}$ is uniquely determined by the stand-off distance. This problem can be overcome by rescaling the model to fit a pre-defined shape of the bow shock and magnetopause. In this study we use a general paraboloidal bow shock model of the form (2), where the "flaring" parameter $b_{\mathrm{bs}}$ can be chosen independently on $R_{\mathrm{bs}}$. Many standard and well tested bow shock models (Filbert and Kellogg, 1979; Farris et al., 1991; Farris and Russell, 1994; Cairns et al., 1995) are formulated or can be easily recast in this form. We used a standard magnetopause model described in Shue et al. (1997), but the method can be easily modified to accommodate other magnetopause shapes.

Let the bow shock be modeled by a paraboloid (Eq. 2) with parameters $R_{\mathrm{bs}}^{(M)}$ and $b_{\mathrm{bs}}^{(M)}$ and let the magnetopause be described by the Shue et al. (1997) model with parameters $R_{\mathrm{mp}}^{(M)}$ and $\alpha_{\mathrm{mp}}^{(M)}$. We will now rescale the KF94 model to match these prescribed boundaries in two steps.

In the first step, the common focus of the paraboloid KF94 models is shifted along the $\mathrm{x}$-axis to make the bow shock 

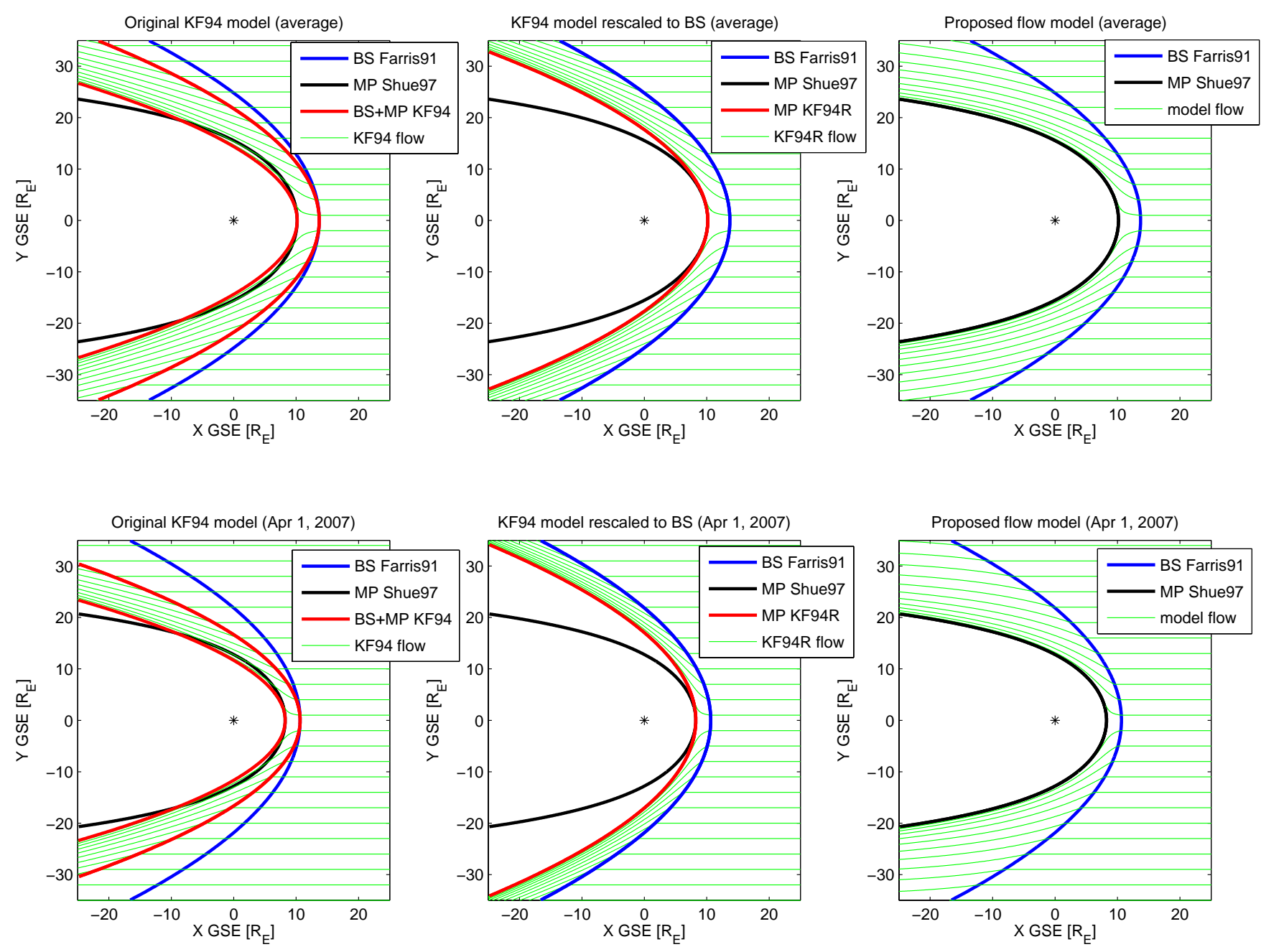

Fig. 2. Comparison of flowlines and boundaries of the model magnetosheath (red line) for the original KF94 model (left), the KF94R model rescaled to fit a general paraboloid bow shock (center) and the final fully rescaled model (right). Superimposed is the Farris et al. (1991) bow shock model (blue) and Shue et al. (1997) magnetopause (black). The top three panels show the comparison for average solar wind parameters, the bottom plots for a set of solar wind parameters from 1 April 2007, 00:00 UT.

model (Eq. 2) coincide with the desired bow shock model. In the KF94 model, the bow shock surface is described by Eq. (2) where the parameter $b_{\mathrm{bs}}$ is fully determined by $R_{\mathrm{bs}}$ and $R_{\mathrm{mp}}$, as $b_{\mathrm{bs}}=1 /\left(4 R_{\mathrm{bs}}-2 R_{\mathrm{mp}}\right)$.

When we introduce a transformation

$$
\begin{aligned}
\boldsymbol{r}_{\mathrm{f}} & =\left[R_{\mathrm{mp}}^{(M)} / 2+\Delta f, 0,0\right] \\
R_{\mathrm{bs}} & =R_{\mathrm{bs}}^{(M)}-\Delta f \\
R_{\mathrm{mp}} & =R_{\mathrm{mp}}^{(M)}-\Delta f
\end{aligned}
$$

to Eq. (2), the focus of paraboloids is shifted by $\Delta f$, but the parameters $R_{\mathrm{bs}}$ and $R_{\mathrm{mp}}$ are corrected to keep the standoff distances of the boundaries from the Earth unchanged. However, the curvature of the paraboloidal surface is modified by this transformation as

$b_{\mathrm{bs}}=1 /\left(4 R_{\mathrm{bs}}-2 R_{\mathrm{mp}}-\Delta f\right)$.
Therefore, the transform (4) with

$$
\Delta f=2 R_{\mathrm{bs}}^{(M)}-R_{\mathrm{mp}}^{(M)}-1 /\left(2 b_{\mathrm{bs}}^{(M)}\right)
$$

can be used to rescale the shock surface to fit arbitrarily chosen parameters $R_{\mathrm{bs}}^{(M)}, R_{\mathrm{mp}}^{(M)}$ and $b_{\mathrm{bs}}^{(M)}$. The rescaling procedure is also illustrated in Fig. 1. The shock and magnetopause surfaces, as well as the flow vector corresponding to the rescaled model, can now be obtained from Eqs. (2)(3) with corrected parameters (4). Hereafter, we refer to this rescaled model as KF94R.

The effect of the rescaling is demonstrated in Fig. 2. The two left panels show a comparison of the KF94 shock and magnetopause (in red) with a paraboloid model bow-shock (Farris et al., 1991) (blue) and a Shue et al. (1997) model (black). Model flowlines are also plotted. The top three plots correspond to average shapes of the boundaries, the bottom 
plots to a more extreme high Mach number event modeled based on solar wind conditions from 1 April 2007, 00:00 UT (from OMNI2 data: $M_{\mathrm{A}}=12.2, \beta=3.7$ ). It is easily seen that both models agree reasonably well in the sub-solar region, but the correspondence gets worse further downstream on the flanks of the magnetosheath and a large fraction of the magnetosheath may not be correctly covered by the KF94 model.

The two plots in the middle column of Fig. 2 show the same comparison for the rescaled KF94R model. After this rescaling, the new KF94R bow shock coincides exactly with the desired paraboloidal bow shock. The KF94R magnetopause is however deformed and deviates from the Shue et al. (1997) model, often more than in the original KF94 implementation.

The problem is remedied in the second step of the rescaling procedure. This step is based on the assumption that the flow velocity vector at a given point in the magnetosheath can be approximated by the velocity at a corresponding point in the model KF94R magnetosheath, at the same angle $\vartheta$ and at the same fractional distance $F$. The latter quantity is defined as

$F(\vartheta)=\frac{r(\vartheta)-r_{\mathrm{bs}}(\vartheta)}{r_{\mathrm{bs}}(\vartheta)-r_{\mathrm{mp}}(\vartheta)}$

and together with angles $\vartheta$ and $\varphi$ forms an alternative curvilinear magnetosheath coordinate system, named "Magnetosheath-Interplanetary Medium" (MIPM) reference frame by Verigin et al. (2006). Here, $r_{\mathrm{bs}}$ and $r_{\mathrm{mp}}$ are the radial shock and magnetopause distance, respectively, in the direction given by $\vartheta$ for the chosen models.

To calculate the flow velocity at a given point $\boldsymbol{r}$ in the magnetosheath, we proceed as follows: (i) The MIPM coordinates $(F, \vartheta, \varphi)$ are calculated using Eqs. (1) and (7) with the chosen models for the bow shock and the magnetopause, in our case Farris et al. (1991) and Shue et al. (1997). (ii) We calculate the corresponding KF94R point $\tilde{\boldsymbol{r}}$ with the same MIPM coordinates, but using $\tilde{r}_{\mathrm{bs}}$ and $\tilde{r}_{\mathrm{mp}}$ from the KF94R model in Eq. (7). (iii) From $\tilde{\boldsymbol{r}}$ is calculated the velocity vector $\tilde{\boldsymbol{v}}$ in the KF94R reference frame using Eq. (3). (iv) The velocity vector $\tilde{\boldsymbol{v}}$ is transformed back from the KF94R magnetosheath to the original GSE reference frame. This last step is more complicated to be tackled analytically, because the transformation is non-linear and depends on the form of the magnetopause model. It is however easily solved approximately by choosing a small time increment $\Delta t$ and calculating the position of an adjacent point on the same flowline $\tilde{\boldsymbol{r}}^{\prime}=\tilde{\boldsymbol{r}}+\tilde{\boldsymbol{v}} \Delta t$. The point $\tilde{\boldsymbol{r}}^{\prime}$ is then easily transformed from the KF94R reference frame to the original GSE frame in a manner analogous to steps 1 and 2 above (let $\boldsymbol{r}^{\prime}$ be the resulting advanced GSE point). The resulting flow velocity vector in GSE coordinates is obtained as $\boldsymbol{v}=\left(\boldsymbol{r}^{\prime}-\boldsymbol{r}\right) / \Delta t$.

The two rightmost plots in Fig. 2 show the final rescaled model. Clearly, both the shock and magnetopause boundaries

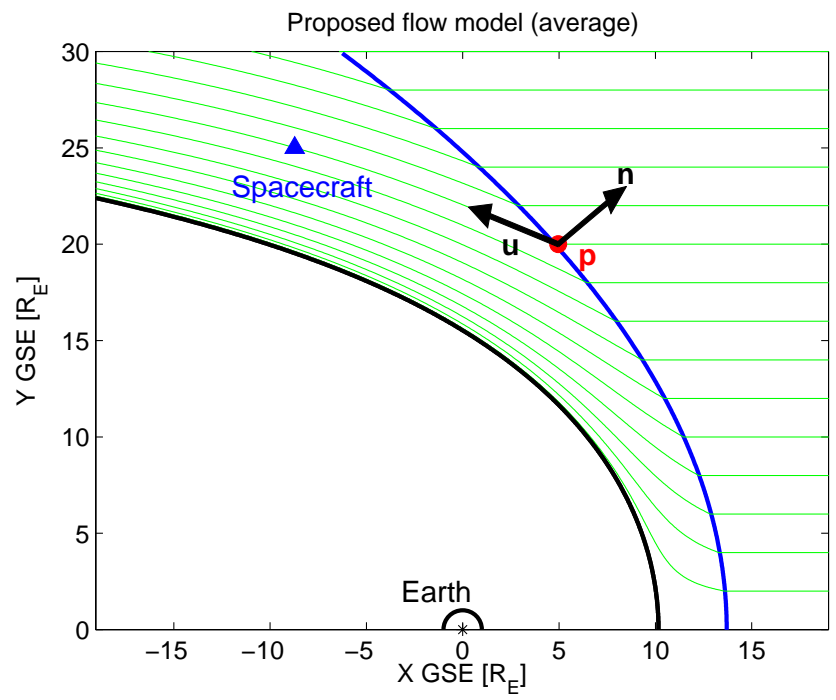

Fig. 3. A schematic representation of back-tracing of the flowline to the bow shock and of the relevant shock parameters used in Sect. 2.2.

fit exactly the desired model shapes, and the flowlines are scaled accordingly.

\subsection{Flow velocity magnitude}

The procedure described in the previous section gives the direction of plasma flow velocity for any point in the magnetosheath. Further effort is necessary to obtain the magnitude of the velocity vector; the above model includes an unknown constant $v_{m 0}$ in Eq. (3) which still needs to be calculated. In this work we follow the approach proposed in Génot et al. (2011), where the analogy between magnetic field and plasma mass flow, $\nabla(\rho \boldsymbol{v})=\nabla \boldsymbol{B}=0$, is exploited further.

For a given point $\boldsymbol{r}$ in the magnetosheath, the above model (where we set $v_{m 0}=1$ ) is used to iteratively trace the flowline back to the bow shock. Let $\boldsymbol{p}$ denote the point where the model flowline passing through $\boldsymbol{r}$ intersects the bow shock, $\boldsymbol{u}$ the unit downstream flow velocity direction vector at point $\boldsymbol{p}$ obtained from the flow model, and $\boldsymbol{n}$ the normal to the bow shock at the same point. These parameters are illustrated in Fig. 3.

Following Génot et al. (2011), we now apply the RankineHugoniot conditions $v_{\mathrm{u}}^{(n)} \rho_{\mathrm{u}}=v_{\mathrm{d}}^{(n)} \rho_{\mathrm{d}}$ and $v_{\mathrm{u}}^{(t)}=v_{\mathrm{d}}^{(t)}$. Here the superscripts $(n)$ and $(t)$ denote the component of the vector parallel and perpendicular to the normal $\boldsymbol{n}$, and subscripts "u" and "d" upstream and downstream parameters, relative to the shock crossing. The solar wind velocity $\boldsymbol{v}_{\mathrm{u}}$ and density $\rho_{\mathrm{u}}$ are considered inputs to the model, and the downstream flow velocity behind the shock is parallel to $\boldsymbol{u}$ and can be expressed as

$\mathbf{v}_{\mathrm{d}}=\frac{v_{\mathrm{u}}^{(n)} \rho_{\mathrm{u}}}{u^{(n)} \rho_{\mathrm{d}}} \boldsymbol{u}=\frac{v_{\mathrm{u}}^{(t)}}{u^{(t)}} \boldsymbol{u}$. 


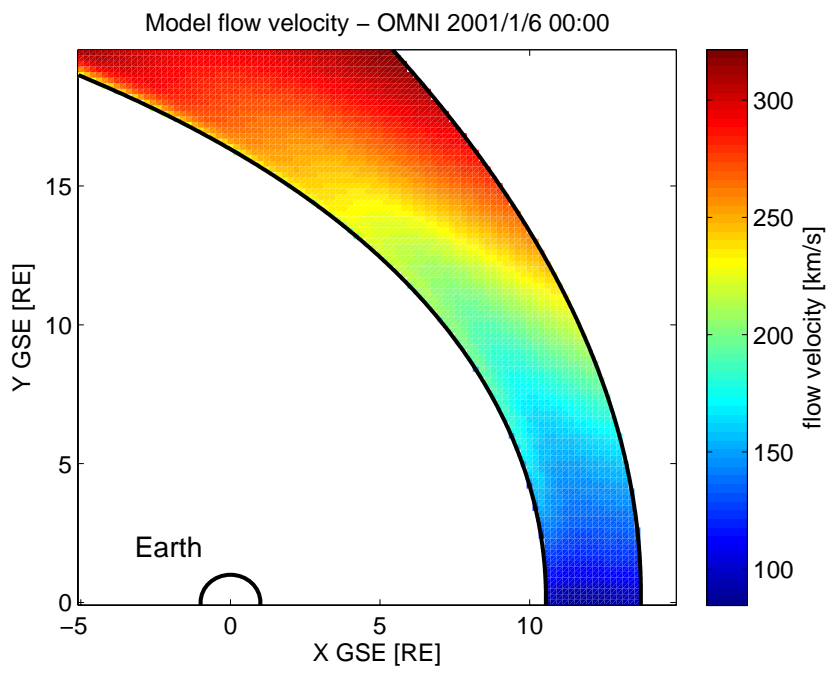

Fig. 4. Color-coded flow speed in GSE equatorial plane calculated from the model. Upstream conditions from 1 June 2001, 00:00 UT were chosen for this example, being close to average solar wind parameters: $V_{\mathrm{u}}=410 \mathrm{~km} \mathrm{~s}^{-1},\left|n_{\mathrm{u}}\right|=4.5 \mathrm{~cm}^{-3},\left|\boldsymbol{B}_{\mathrm{u}}\right|=$ $7.2 \mathrm{nT}, M_{\mathrm{A}}=6.1$, and $\beta=0.5$.

Note that the coefficient before $\boldsymbol{u}$ is a positive number, since the normal component of upstream and downstream flow velocity must have the same sign and density is a positive number. The ratio of upstream to downstream plasma density across the shock can be expressed from the same equations as

$\rho_{\mathrm{d}} / \rho_{\mathrm{u}}=u^{(t)} v_{\mathrm{u}}^{(n)} / u^{(n)} v_{\mathrm{u}}^{(t)}$.

If we assume that plasma density is constant along the flowline from the shock to the point $\boldsymbol{r}$, the constant $v_{m 0}$ is easily calculated from Eq. (8). While the approximation of constant density works reasonably well, Génot et al. (2011) proposed use of an ad hoc model for magnetosheath density which introduces a moderate density decrease near the magnetopause (associated with plasma depletion layer). Even though the model is relatively crude, it seems to correspond slightly better with observations (see Sect. 3.2) and is easy to implement. Similarly to Génot et al. (2011), we thus assume that density $\rho$ at a fractional distance $F$ can be estimated from the density on the same flowline near the shock $\rho_{\mathrm{d}}$ as

$\rho / \rho_{\mathrm{d}}=0.8+0.2 \times \tanh (4 F)$.

From the continuity equation and Eq. (8) we finally obtain the formula for the magnetosheath flow velocity (and for $\left.v_{m 0}\right)$ :

$\boldsymbol{v}=\frac{v_{\mathrm{u}}^{(t)} \rho_{\mathrm{d}}}{u^{(t)} \rho} \boldsymbol{u}=v_{m 0} \boldsymbol{u}$

Here, it should be noted that the choice of the tangential direction $(t)$ in the plane tangential to the shock is arbitrary. To minimize the effect of measurement errors, we choose two orthogonal directions in the tangential plane, calculate the $v_{\mathrm{u}}^{(t)} / u^{(t)}$ for both and use a quadratic mean value in Eq. (11).

In this section we introduced a magnetosheath velocity model, which allows predicting the plasma bulk flow velocity for any location in the magnetosheath using only the upstream solar wind parameters (namely the solar wind velocity vector and parameters required by shock and magnetopause models: the IMF vector and plasma density). Flow vector direction can be calculated directly by a simple formula. To obtain the flow velocity magnitude, the flowline needs to be iteratively back-traced to the bow shock.

\section{Validation of the model}

Since the magnetosheath model presented in the previous section includes a number of assumptions and some ad hoc steps, the model needs to be tested against observed data and its performance evaluated. As a first check of the validity of the model, we plotted a color-coded magnitude of flow velocity in Fig. 4 in XY GSE plane. This plot can be directly compared to similar figures in the literature (Génot et al., 2011; Spreiter et al., 1966) and shows a good qualitative agreement.

To obtain a more quantitative assessment of the model performance, in this section we further test the model on in situ plasma observations from Cluster (Escoubet et al., 2001) and THEMIS spacecraft (Angelopoulos, 2008) and compare the results with the Génot et al. (2011) model.

\subsection{Dataset}

Our test dataset is composed of in situ measurements of magnetosheath ion flow velocity and corresponding sets of upstream solar wind parameters. Specifically:

- Ion flow velocity from the HIA instrument (Rème et al., 1997) on the Cluster 1 spacecraft. We used calibrated on-board calculated moments obtained from the Cluster Active Archive. Measurements from Cluster magnetosheath crossings between 4 November 2007 and 10 June 2008 were included. This particular interval was chosen because of a significant overlap with the first year of THEMIS magnetosheath measurements.

- Ion flow velocity from the ESA instrument on the THEMIS TH-B (P1) and TH-C (P2) spacecraft (McFadden et al., 2008). We used on-board calculated moments (MOM data product). Measurements from THEMIS magnetosheath crossings between 29 October 2007 and 5 December 2007 and between 18 April 2008 and 12 June 2008 were included.

- OMNI2 solar wind parameters propagated to the bow shock at $1 \mathrm{~min}$ time resolution (King and Papitashvili, 2005). We used the plasma flow velocity, plasma 

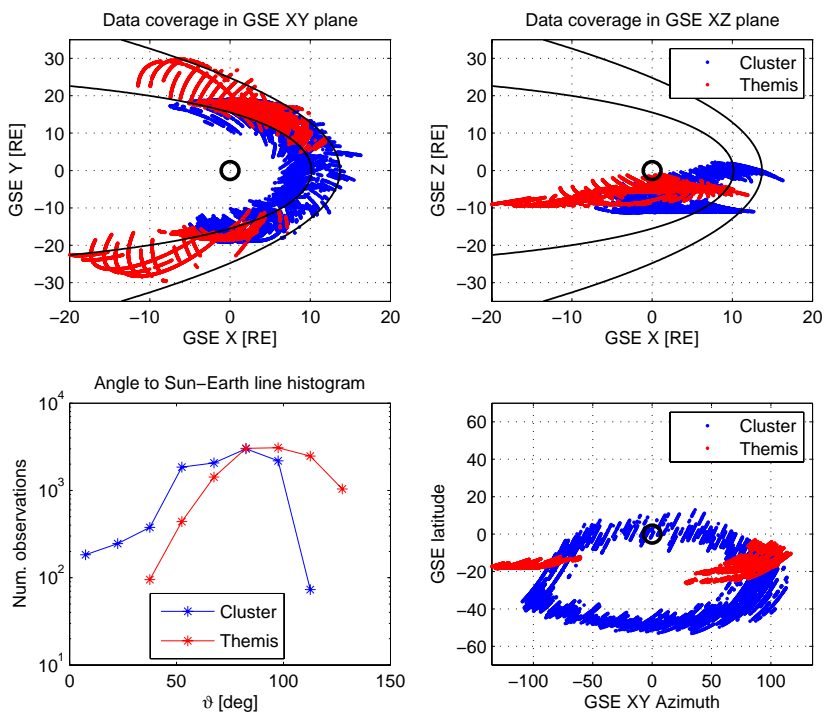

Fig. 5. Coverage of the magnetosheath by Cluster and THEMIS test datasets. The bottom left panel shows the histogram of data points binned in the zenith angle $\vartheta$. The dataset contains a total of 23554 observations: 11403 from Cluster 1 and 12151 from THEMIS P1 (TH-B) and P2 (TH-C).

density, solar wind magnetic field and bow shock nose location parameters from the OMNI2 dataset.

For the ion velocity measurements, each datapoint represents a 5-min average. Only measurements taken in an instrument mode appropriate for magnetosheath plasma observations were included. Datapoints with an incomplete set of solar wind parameters in the OMNI dataset were excluded. Bow shock and magnetopause crossings were identified by visual inspection of magnetic field and ion data, and only intervals demonstrating magnetic field and plasma signatures consistent with magnetosheath were included in the dataset. The final dataset contains 23554 valid magnetosheath velocity measurements (11403 from Cluster and 12151 from THEMIS) and covers a period of 7.5 months.

The distribution of the data points in GSE coordinates is shown in Fig. 5. It can be seen that the dataset covers a wide range of zenith angles $\vartheta$ from the sub-solar point to magnetosheath flanks (down to $x \approx-20 R_{\mathrm{E}}$ ). The measurements are distributed mostly south from the equatorial plane at latitudes from 0 to $-50^{\circ}$ due to the orbits of Cluster and THEMIS spacecraft in the studied period. The asymmetric distribution in latitude might introduce certain bias in the statistics, but no fitting to data was employed in the derivation of the model and this bias could only result in underestimating of the discrepancy between the model and data. Since the model is cylindrically symmetric and so is (to the first order) the magnetosheath flow outside magnetospheric cusps, we do not see a reason why the accuracy of model prediction should be significantly different in the Northern Hemisphere. Two different spacecraft with different orbits and instrumen-
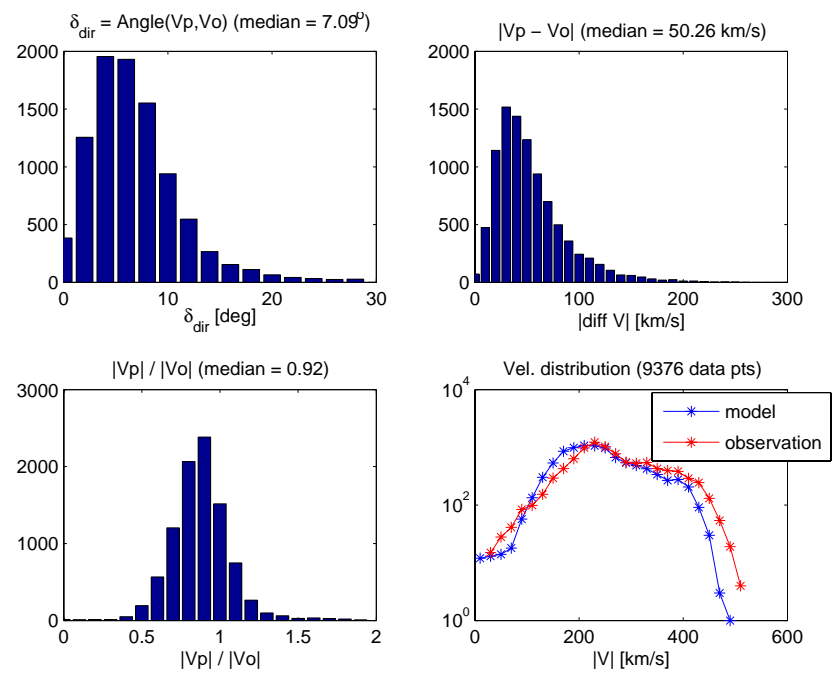

Fig. 6. Comparison of the predicted flow velocity with Cluster observations. Top left: histogram of the angle $\delta_{\text {dir }}$ between model and observed velocities. Top right: histogram of the magnitude of vector difference $\left|\boldsymbol{V}_{\mathrm{p}}-\boldsymbol{V}_{\mathrm{o}}\right|$. Bottom left: histogram of the ratio of magnitudes $V_{\mathrm{p}} / V_{\mathrm{o}}$. Bottom right: distribution of predicted and observed velocity magnitudes $V_{\mathrm{p}}$ (blue) and $V_{\mathrm{o}}$ (red).

tation are included to minimize instrument-specific systematic effects in the dataset.

\subsection{Comparison with Génot et al. (2011) model}

The statistical dataset described in the previous section was used to compare the prediction of our model to observed data and evaluate the relative performance of our model and the model described in Génot et al. (2011).

The results are summarized in Table 1, where several parameters characterizing the deviation of the model prediction from observed values are presented. The number of observations in each dataset that fall outside the model magnetosheath boundaries is given to characterize the applicability of the models to a real magnetosheath (the "Points outside model MSHT" rows in the table). For these observations the respective model cannot be used. For points within model magnetosheath, the error in the predicted flow velocity direction (which can be calculated efficiently without the need for flowline tracing) and the predicted magnitude are evaluated separately. The statistical results are shown for a total dataset, for datasets from individual spacecraft, and for subsolar (zenith angle $\left.\vartheta \leq 75^{\circ}\right)$ and flank $\left(\vartheta>75^{\circ}\right)$ restricted datasets.

It is readily seen from Table 1 that significantly more observations fall outside the model magnetosheath in the case of the Génot et al. (2011) model. The rescaling and modification of the KF94 model proposed in the present article thus improves the applicability of the model. For the points where the models can be used, the discrepancies between model prediction and observations are comparable. The error 
Table 1. Comparison of the performance of the proposed model with the model of Génot et al. (2011) on the datasets. Here $\delta_{\text {dir }}$ is the angle between the predicted velocity $\boldsymbol{V}_{\mathrm{p}}$ and observed flow velocity $\boldsymbol{V}_{\mathrm{o}}$. The quality of velocity magnitude prediction is quantified by the $V_{\mathrm{p}} / V_{\mathrm{o}}$ ratio. For both quantities the table shows a median value and number, absolute and relative, of data points where the prediction deviates by less than $10^{\circ}$ in direction or by less than $25 \%$ in magnitude from the observation. We also give the number of points in each dataset which fall outside the model magnetosheath, and thus do not allow the application of the respective flow model.

\begin{tabular}{|c|c|c|c|c|c|}
\hline & All & Cluster & Themis & $\vartheta \leq 75^{\circ}$ & $\vartheta>75^{\circ}$ \\
\hline Number of data points & 23554 & 11403 & 12151 & 7434 & 16120 \\
\hline \multicolumn{6}{|l|}{ Proposed model } \\
\hline Points outside model MSHT & $2415(10.3 \%)$ & $1713(15.0 \%)$ & $702(5.8 \%)$ & $1266(17.0 \%)$ & $1149(7.1 \%)$ \\
\hline$\delta_{\text {dir }}($ median $)$ & $6.6^{\circ}$ & $7.1^{\circ}$ & $6.2^{\circ}$ & $7.0^{\circ}$ & $6.5^{\circ}$ \\
\hline$\delta_{\mathrm{dir}}<10^{\circ}(\%)$ & $16821(80.8 \%)$ & $7079(75.5 \%)$ & $9742(85.1 \%)$ & $4234(72.3 \%)$ & $12587(84.1 \%)$ \\
\hline$V_{\mathrm{p}} / V_{\mathrm{o}}($ median $)$ & 0.94 & 0.92 & 0.96 & 0.97 & 0.93 \\
\hline$\left|V_{\mathrm{p}} / V_{\mathrm{o}}-1\right|<0.25(\%)$ & $18012(86.5 \%)$ & $7621(81.3 \%)$ & $10391(90.8 \%)$ & $4715(80.5 \%)$ & $13297(88.8 \%)$ \\
\hline \multicolumn{6}{|l|}{ Génot et al. (2011) model } \\
\hline Points outside model MSHT & $5975(25.4 \%)$ & $2648(23.2 \%)$ & $3327(27.4 \%)$ & $1946(26.2 \%)$ & $4029(25.0 \%)$ \\
\hline$\delta_{\text {dir }}($ median $)$ & $6.1^{\circ}$ & $7.8^{\circ}$ & $4.8^{\circ}$ & $9.5^{\circ}$ & $5.1^{\circ}$ \\
\hline$\delta_{\operatorname{dir}}<10^{\circ}(\%)$ & $13622(77.5 \%)$ & $5836(66.7 \%)$ & $7786(88.2 \%)$ & $2943(53.6 \%)$ & $10679(88.3 \%)$ \\
\hline$V_{\mathrm{p}} / V_{\mathrm{o}}($ median $)$ & 1.13 & 1.14 & 1.12 & 1.25 & 1.10 \\
\hline$\left|V_{\mathrm{p}} / V_{\mathrm{o}}-1\right|<0.25(\%)$ & $13429(76.4 \%)$ & $5924(67.7 \%)$ & $7505(85.1 \%)$ & $4715(49.1 \%)$ & $10734(88.7 \%)$ \\
\hline
\end{tabular}
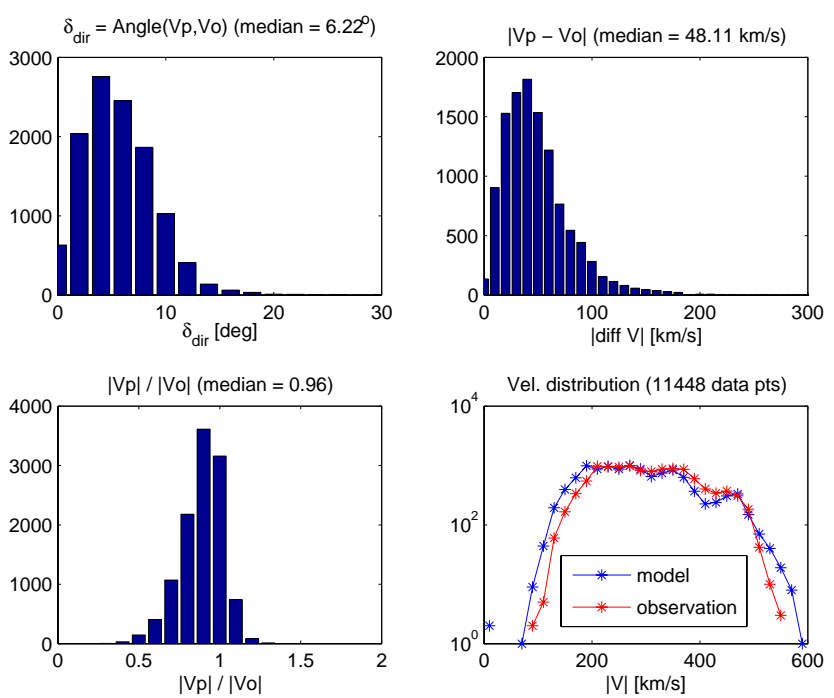

Fig. 7. Comparison of the predicted flow velocity with THEMIS $\mathrm{P} 1$ and P2 observations. The content of the panels is analogous to Fig. 6.

in the model direction is in general very small (median error is less than $7^{\circ}$ ) and of similar magnitude for both models. The magnitude of flow velocity is slightly underestimated by the proposed model (median $V_{\mathrm{p}}=0.94 V_{\mathrm{o}}$ ) and overestimated by the Génot et al. (2011) model (median $V_{\mathrm{p}}=1.13 V_{\mathrm{o}}$ ).

Both models give better predictions on the flanks of the magnetosheath and consequently for the THEMIS dataset which contains more flank observations. In fact, for the THEMIS dataset, the predictions of Génot et al. (2011), in the cases where it could be used, are slightly better than for our model. It must be noted, however, that in the case of the flank dataset, the Génot et al. (2011) model was inapplicable for $25.0 \%$ of observations. For comparison, our model could not be used for only $5.8 \%$ of points in the dataset. A similar discrepancy is found for the THEMIS dataset. The correspondence of the model velocity direction with observations was in general worse for the sub-solar dataset where the proposed model shows a significant improvement over the Génot et al. (2011) model. The prediction of velocity magnitude was on average better for the proposed model for all test datasets, again with a greater improvement in the sub-solar magnetosheath.

The comparison of the proposed model with the data is visualized in Fig. 6 for the Cluster dataset and in Fig. 7 for the THEMIS dataset. The plots illustrate and confirm the good agreement of the model with observations and better performance of the model on the THEMIS dataset. The plots also show the distribution of very large errors: large errors in the predicted magnitude usually correspond to underestimated velocity, and the prediction is in general worse for very large or very small flow velocities.

\section{Discussion and conclusions}

The results presented in the previous section clearly demonstrate that the model proposed in this article provides estimates of plasma flow velocity in the magnetosheath in excellent correspondence with Cluster and THEMIS observations. The model is relatively simple to implement: the flow velocity direction (often sufficient) can be calculated directly 
using an algebraic formula, and the velocity magnitude estimate can be obtained at an additional cost of performing an iterative flowline tracing procedure. In comparison with the previous models by Kobel and Flückiger (1994) and Génot et al. (2011), our model has fewer restrictions on the shape of the model bow shock and magnetopause and in general provides better correspondence with observations.

The correspondence of the flow velocity with observations was found to be exceptionally good and on par with the instrumental error associated with Cluster and THEMIS particle instruments. For the Cluster CIS HIA instrument, this error is typically around $6^{\circ}$ under optimal conditions when sufficient count rates are registered by the sensor without saturation (Rème et al., 1997; Martz et al., 1993). The method used for the flow velocity magnitude calculation uses an ad hoc model of plasma density profile, and it could most likely be improved if required by a specific application. The accuracy of the prediction is strongly dependent on the bow shock and magnetopause models serving as inputs to the flow velocity model. We chose standard models with overall good performance for a wide range of upstream conditions, latitudes and longitudes. For specific applications, a user may choose different models to obtain better performance.

Another point where the present model could be improved is the modeling of the bow shock transition. In our approach, we used greatly simplified forms of Rankine-Hugoniot conditions, shown to be adequate for our purpose by Génot et al. (2011). A more sophisticated solution for the downstream flow velocity, compatible with the respective shock model, could be included in the flow model, possibly improving the prediction of the flow velocity magnitude. Particularly viable models would be those described in Verigin et al. (2003) and Petrinec and Russell (1997).

The model could be easily applied to other magnetized planets, provided that good bow shock and magnetopause models for the given planet were available. The model would have to be modified to incorporate those models, and since the solar wind and magnetospheric plasma conditions, as well as field geometry, can be very different for other planets, the ad hoc density model (Eq. 10) used in this paper may need to be modified as well. The quality of the prediction for those cases may be worse than for the terrestrial magnetosheath, and the model would have to be tested against observed data accordingly.

We believe that the model, as presented, is sufficiently robust and general for most magnetosheath studies requiring the knowledge of magnetosheath flowlines for the purpose of tracing the flow between multiple observation points or from a given spacecraft location to the bow shock, and it will present a computationally cheaper alternative to the models based on gas-dynamic equations (Stahara, 2002).

Acknowledgements. We thank the Cluster principal investigators E. Lucek and I. Dandouras and their instrument teams for the data used in this study. We appreciate the Cluster Active Archive facility, enabling an easy centralized access to full resolution calibrated Cluster data. JS acknowledges the support of the grant P209/12/2394 of the Grant Agency of Czech Republic. We acknowledge NASA contract NAS5-02099 and V. Angelopoulos for use of data from the THEMIS Mission. Specifically: C. W. Carlson and J. P. McFadden for use of ESA data and K. H. Glassmeier, U. Auster and W. Baumjohann for the use of FGM data provided under the lead of the Technical University of Braunschweig and with financial support through the German Ministry for Economy and Technology and the German Center for Aviation and Space (DLR) under contract 50 OC 0302. The OMNI2 data were obtained from the GSFC/SPDF OMNIWeb interface at http://omniweb.gsfc.nasa.gov; we appreciate the effort of the OMNI team at GSFC/SPDF as well as of the instrument teams contributing spacecraft data to the OMNI database.

Topical Editor I. A. Daglis thanks two anonymous referees for their help in evaluating this paper.

\section{References}

Angelopoulos, V.: The THEMIS Mission, Space Sci. Rev., 141, 534, doi:10.1007/s11214-008-9336-1, 2008.

Cairns, I. H., Fairfield, D. H., Anderson, R. R., Carlton, V. E. H., Paularena, K. I., and Lazarus, A. J.: Unusual locations of Earth's bow shock on September 24-25, 1987: Mach number effects, J. Geophys. Res., 100, 47-62, doi:10.1029/94JA01978, 1995.

Escoubet, C. P., Fehringer, M., and Goldstein, M.: Introduction: The Cluster mission, Ann. Geophys., 19, 1197-1200, doi:10.5194/angeo-19-1197-2001, 2001.

Farris, M. H. and Russell, C. T.: Determining the standoff distance of the bow shock: Mach number dependence and use of models, J. Geophys. Res., 99, 17681, doi:10.1029/94JA01020, 1994.

Farris, M. H., Petrinec, S. M., and Russell, C. T.: The thickness of the magnetosheath - Constraints on the polytropic index, Geophys. Res. Lett., 18, 1821-1824, 1991.

Filbert, P. C. and Kellogg, P. J.: Electrostatic noise at the plasma frequency beyond the earth's bow shock, J. Geophys. Res., 84, 1369-1381, 1979.

Génot, V., Broussillou, L., Budnik, E., Hellinger, P., Trávníček, P. M., Lucek, E., and Dandouras, I.: Timing mirror structures observed by Cluster with a magnetosheath flow model, Ann. Geophys., 29, 1849-1860, doi:10.5194/angeo-29-1849-2011, 2011.

Hayosh, M., Šafránková, J., Němeček, Z., Prech, L., Kudela, K., and Zastenker, G. N.: Relationship between high-energy particles and ion flux in the magnetosheath, Planet. Space Sci., 53, 103-115, doi:10.1016/j.pss.2004.09.034, 2005.

Kallio, E. J. and Koskinen, H. E. J.: A semiempirical magnetosheath model to analyze the solar wind-magnetosphere interaction, J. Geophys. Res., 105, 27469-27480, doi:10.1029/2000JA900086, 2000.

King, J. H. and Papitashvili, N. E.: Solar wind spatial scales in and comparisons of hourly Wind and ACE plasma and magnetic field data, J. Geophys. Res., 110, A02104, doi:10.1029/2004JA010649, 2005.

Kobel, E. and Flückiger, E. O.: A model of the steady state magnetic field in the magnetosheath, J. Geophys. Res., 99, 23617-23622, doi:10.1029/94JA01778, 1994. 
Martz, C., Sauvaud, J. A., and Rème, H.: Accuracy of ion distribution measurements and related parameters using the Cluster CIS experiment, in: Spatio-Temporal Analysis for Resolving Plasma Turbulence (START), edited by: Escoubet, C. P., vol. WPP-047, pp. 229-236, European Space Agency, 1993.

McFadden, J. P., Carlson, C. W., Larson, D., Ludlam, M., Abiad, R., Elliott, B., Turin, P., Marckwordt, M., and Angelopoulos, V.: The THEMIS ESA Plasma Instrument and In-flight Calibration, Space Sci. Rev., 141, 277-302, doi:10.1007/s11214-008-9440-2, 2008.

Petrinec, S. M. and Russell, C. T.: Hydrodynamic and MHD Equations across the Bow Shock and Along the Surfaces of Planetary Obstacles, Space Sci. Rev., 79, 757-791, doi:10.1023/A:1004938724300, 1997.

Rème, H., Bosqued, J. M., Sauvaud, J. A., Cros, A., Dandouras, J., Aoustin, C., Bouyssou, J., Camus, T., Cuvilo, J., Martz, C., Medale, J. L., Perrier, H., Romefort, D., Rouzaud, J., D’Uston, C., Mobius, E., Crocker, K., Granoff, M., Kistler, L. M., Popecki, M., Hovestadt, D., Klecker, B., Paschmann, G., Scholer, M., Carlson, C. W., Curtis, D. W., Lin, R. P., McFadden, J. P., Formisano, V., Amata, E., Bavassano-Cattaneo, M. B., Baldetti, P., Belluci, G., Bruno, R., Chionchio, G., di Lellis, A., Shelley, E. G., Ghielmetti, A. G., Lennartsson, W., Korth, A., Rosenbauer, H., Lundin, R., Olsen, S., Parks, G. K., McCarthy, M., and Balsiger, H.: The Cluster Ion Spectrometry (CIS) Experiment, Space Sci. Rev., 79, 303-350, doi:10.1023/A:1004929816409, 1997.

Romashets, E. P., Poedts, S., and Vandas, M.: Modeling of the magnetic field in the magnetosheath region, J. Geophys. Res. (Space Physics), 113, A02203, doi:10.1029/2006JA012072, 2008.

Russell, C. T., Luhmann, J. G., Odera, T. J., and Stuart, W. F.: The rate of occurrence of dayside Pc 3,4 pulsations - The L-value dependence of the IMF cone angle effect, Geophys. Res. Lett., 10, 663-666, doi:10.1029/GL010i008p00663, 1983.

Shue, J.-H., Chao, J. K., Fu, H. C., Russell, C. T., Song, P., Khurana, K. K., and Singer, H. J.: A new functional form to study the solar wind control of the magnetopause size and shape, J. Geophys. Res., 102, 9497-9512, doi:10.1029/97JA00196, 1997.

Song, P., Russell, C. T., Gombosi, T. I., Spreiter, J. R., Stahara, S. S., and Zhang, X. X.: On the processes in the terrestrial magnetosheath 1. Scheme development, J. Geophys. Res., 104, 22345 22356, doi:10.1029/1999JA900247, 1999a.

Song, P., Russell, C. T., Zhang, X. X., Stahara, S. S., Spreiter, J. R., and Gombosi, T. I.: On the processes in the terrestrial magnetosheath 2. Case study, J. Geophys. Res., 104, 22357-22374, doi:10.1029/1999JA900246, 1999b.
Spreiter, J. R. and Alksne, A. Y.: Comparison of theoretical predictions of the flow and magnetic fields exterior to the magnetosphere with the observations of Pioneer 6, Planet. Space Sci., 16, 971-979, doi:10.1016/0032-0633(68)90013-5, 1968.

Spreiter, J. R. and Stahara, S. S.: A new predictive model for determining solar wind-terrestrial planet interactions, J. Geophys. Res., 85, 6769-6777, doi:10.1029/JA085iA12p06769, 1980.

Spreiter, J. R. and Stahara, S. S.: Gasdynamic and magnetohydrodynamic modeling of the magnetosheath: A tutorial, Adv. Space Res., 14, 5-19, doi:10.1016/0273-1177(94)90042-6, 1994.

Spreiter, J. R., Summers, A. L., and Alksne, A. Y.: Hydromagnetic flow around the magnetosphere, Planet. Space Sci., 14, 223 , doi:10.1016/0032-0633(66)90124-3, 1966.

Spreiter, J. R., Alksne, A. Y., and Summers, A. L.: External Aerodynamics of the Magnetosphere, in: Physics of the Magnetosphere, edited by: Carovillano, R. D. L. and McClay, J. F., vol. 10 of Astrophysics and Space Science Library, pp. 304-378, 1968.

Stahara, S. S.: Adventures in the magnetosheath: two decades of modeling and planetary applications of the Spreiter magnetosheath model, Planet. Space Sci., 50, 421-442, doi:10.1016/S0032-0633(02)00023-5, 2002.

Stahara, S. S., Rachiele, R. R., Molvik, G. A., and Spreiter, J. R.: Development of a preliminary solar wind transport magnetosheath forecast model, NASA STI/Recon Technical Report, PL-TR-93-2169, 1993.

Tátrallyay, M. and Erdős, G.: The evolution of mirror mode fluctuations in the terrestrial magnetosheath, Planet. Space Sci., 50 , 593-599, 2002.

Tátrallyay, M., Erdős, G., Balogh, A., and Dandouras, I.: The evolution of mirror type magnetic fluctuations in the magnetosheath based on multipoint observations, Adv. Space Res., 41, 15371544, doi:10.1016/j.asr.2007.03.039, 2008.

Verigin, M., Slavin, J., Szabo, A., Gombosi, T., Kotova, G., Plochova, O., Szegö, K., Tátrallyay, M., Kabin, K., and Shugaev, F.: Planetary bow shocks: Gasdynamic analytic approach, J. Geophys. Res., 108, 1323, doi:10.1029/2002JA009711, 2003.

Verigin, M. I., Tátrallyay, M., Erdős, G., and Kotova, G. A.: Magnetosheath Interplanetary medium reference frame: Application for a statistical study of mirror type waves in the terrestrial plasma environment, Adv. Space Res., 37, 515-521, doi:10.1016/j.asr.2005.03.042, 2006. 